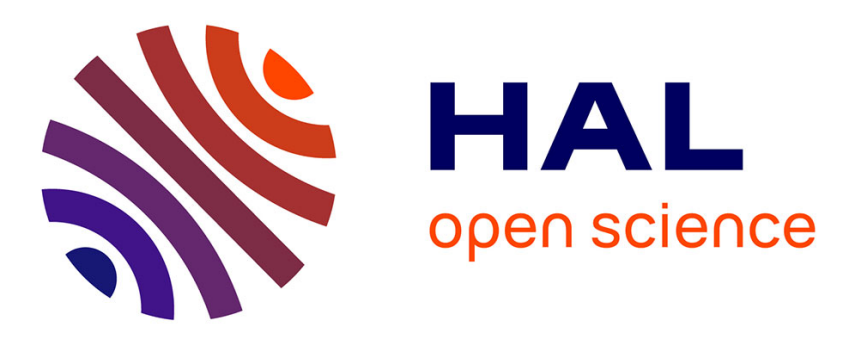

\title{
Probabilistic Observers for a Class of Uncertain Biological Processes
}

Benoit Chachuat, Olivier Bernard

\section{To cite this version:}

Benoit Chachuat, Olivier Bernard. Probabilistic Observers for a Class of Uncertain Biological Processes. International Journal of Robust and Nonlinear Control, 2005, 16, pp.157-171. inria-00122551

\section{HAL Id: inria-00122551 https://hal.inria.fr/inria-00122551}

Submitted on 3 Jan 2007

HAL is a multi-disciplinary open access archive for the deposit and dissemination of scientific research documents, whether they are published or not. The documents may come from teaching and research institutions in France or abroad, or from public or private research centers.
L'archive ouverte pluridisciplinaire HAL, est destinée au dépôt et à la diffusion de documents scientifiques de niveau recherche, publiés ou non, émanant des établissements d'enseignement et de recherche français ou étrangers, des laboratoires publics ou privés. 


\title{
Probabilistic Observers for a Class of Uncertain Biological Processes
}

\author{
B. Chachuat ${ }^{1, \dagger}$ and O. Bernard ${ }^{2, *}$ \\ 1 MIT, Department of Chemical Engineering, 77 Massachusetts Avenue, Cambridge, MA 02139-4307, USA \\ (email: bchachua@mit.edu). \\ 2 INRIA Comore, 2004 route des Lucioles, B.P. 93, 06902 Sophia-Antipolis, France (email: \\ Olivier.Bernard@inria.fr).
}

\begin{abstract}
SUMMARY
In this paper, probabilistic observers are considered for a class of continuous biological processes described by mass-balance-based models. It is assumed that the probability density functions (PDFs) of the uncertain parameters and inputs of the model, as well as the PDFs of the missing initial conditions are known. Then, the PDFs of the unmeasured state variables are obtained, at any time, by considering the image of these initial PDFs by the flow of the dynamic model (differential system). In comparison to classical open-loop asymptotic and interval observers, the method provides information on the confidence level of the estimates rather than simple upper and lower bounds. Moreover, unlike Kalman filters, probabilistic observers are not restricted to Gaussian distributions for the uncertain parameters. The design and application of a probabilistic observer to an industrial wastewater treatment plant is presented. Finally, a number of practical considerations is discussed in connection to both implementation and utilization issues. Copyright (c) 2005 John Wiley \& Sons, Ltd.
\end{abstract}

KEY WORDS: observers; unknown inputs; uncertain parameters; bioprocesses; wastewater treatment; anaerobic digestion

\section{INTRODUCTION}

One of the main difficulties in the monitoring and control of biological reactors lies in the absence, in most applications, of cheap and reliable sensors capable of providing direct, online measurements of the biological state variables. The design of state observers, also called software sensors, for the online monitoring of the state variables which are not measurable in real time has thus received much attention in the literature, and has given rise to many practical applications $[1,2]$.

*Correspondence to: INRIA Comore, 2004 Route des Lucioles, B.P. 93, 06902 Sophia-Antipolis, France. †Presently at: Swiss Federal Institue of Technology (EPFL), Laboratoire d'Automatique, Station 9, CH-1015 Lausanne, Switzerland.

Copyright (c) 2005 John Wiley \& Sons, Ltd. 
Biological processes typically exhibit large uncertainties, which make it difficult to apply classical observation techniques. This is the case in particular for the class of high gain observers $[3,4]$ that generally lack of robustness when dealing with noisy and/or low frequency measurements, as well as parameter uncertainties. More reliable estimates can be obtained by combining the aforementioned high gain observers with Kalman filtering techniques [5, 6], thus yielding extended Kalman filters. Such observers have demonstrated improved ability to cope with measurement noise, and successful applications have even been reported for the estimation of biological systems [7]. However, the large uncertainties resulting from model mismatch as well as the inherent variability of biological processes are not really handled by these approaches that are sensitive to the picking phenomenon [8]. This may also explain why practical applications of high gain observers to full-scale biological processes are scarce.

For biological processes, another approach has been popularized by Bastin and Dochain [1]. It relies on the underlying structure of many bioprocess models that consist of two parts: $(i)$ a linear part based on mass-balance considerations; and ( $i i)$ a nonlinear term which describes the biological reaction rates (kinetics). It should be noted that this latter term refers to the core of the biological activity and is often very sensitive to medium growth conditions; moreover, the associated kinetic parameters are not known accurately in practice. These considerations have motivated the approach proposed by [1] that takes advantage of a linear change of variable to eliminate the uncertain kinetic terms from the observer design; the resulting observers were termed asymptotic observers, and have been widely used in practice [9, 2]. Note that the idea of performing the estimation without knowing the reaction rates has its roots in the notion of reaction invariants $[10,11,12]$ and constitutes an extension of mass-conservation principles. Since they are mainly based on mass-conservation principles (assuming known and constant biological stoichiometry), asymptotic observers are most efficient when the influent composition is well known. In the counterpart however, the corresponding state predictions may converge very slowly; moreover, the estimates may be corrupted in the presence of large measurement noise. In response to this, some authors have proposed a way of combining the convergence and filtering advantages of the high gain approach together with the robustness of the asymptotic observers. For such observers, the state estimates first reach a neighborhood of the unmeasured states very rapidly, from which neighborhood the estimates then converge more slowly, but also more accurately, towards the true values of the state variables [13, 14].

More recently, interval observers have been proposed as a rigorous way of dealing with uncertain processes $[15,16]$. The idea is to estimate bounds enclosing the state variables, provided that conservative bounds are known for the uncertainties (influent composition, model parameters, etc.). The derivation of interval observers requires that the observation principle be weakened in the sense that the observation error is no longer expected to converge to zero asymptotically. Several successful applications have been reported in the field of biological systems (e.g., [17]). But although interval observers are more robust than the aforementioned asymptotic observers, still limited information is obtained with respect to the unmeasured state variables, since "only" an upper bound and a lower bound are derived and these bounds may be weak.

In many situations however, more information than simple upper and lower bounds is known for the uncertainties. In particular, an estimate of their probability density functions (PDFs) is often available, e.g., when the uncertain parameters have been obtained from the application of a parameter identification procedure. In this work, we propose to exploit this additional information, in conjunction to the two-part structure of mass-balance base bioprocess models, 
for estimating the PDFs of the unmeasured state variables. Our approach is based on the same reaction invariants [12] as those used by [1] to formulate the asymptotic observers. We first estimate the PDFs of a set of auxiliary variables, and then deduce the PDFs of the unmeasured part of the state by combining these former PDFs with those of the measured state variables. Loosely speaking, these observers can therefore be seen as a hybrid combination between a (linear) Kalman filter and an asymptotic observer. Moreover, because they provide an estimate of the PDFs of the state variables, the term probabilistic observer has been coined for these observers.

The remainder of the paper is organized as follow. Some results on mass-balance bioprocess modeling and asymptotic observers are first recalled in section 2. Probabilistic observers are then described in section 3 for a class of continuous bioprocesses, and their numerical implementation is closely considered. The method is illustrated in section 4 with the application of probabilistic observer to the estimation of the substrate concentration in an industrial wastewater treatment plant. Finally, a number of practical considerations regarding the use of probabilitic observers are discussed in section 5 .

\section{PRELIMINARIES}

Continuous bioprocesses that are operated in stirred tank bioreactors are often described by means of general mass-balance-based models of the following form [1]:

$$
\dot{\boldsymbol{\xi}}=\mathrm{Kr}(\boldsymbol{\xi})+D\left(\boldsymbol{\xi}^{i n}-\boldsymbol{\xi}\right)-\mathbf{q}(\boldsymbol{\xi})
$$

where $\boldsymbol{\xi} \in \mathbb{R}^{n_{\xi}}$ (resp. $\boldsymbol{\xi}^{i n}$ ) denotes the concentration vector in the liquid phase (resp. in the influent), $\mathrm{K} \in \mathbb{R}^{n_{\xi} \times n_{r}}$ the yield coefficient matrix, $\mathbf{r} \in \mathbb{R}^{n_{r}}$ the reaction rate vector, $\mathbf{q} \in \mathbb{R}^{n_{\xi}}$ the gaseous exchange vector, and $D \in \mathbb{R}$ the dilution rate.

Throughout the paper, we assume that $\left(n_{\xi}-n_{b}\right)$ components of the state variables are measured on-line and we denote by $\boldsymbol{\xi}_{a} \in \mathbb{R}^{n_{\xi}-n_{b}}$ and $\boldsymbol{\xi}_{b} \in \mathbb{R}^{n_{b}}$ the measured and unmeasured state variables, respectively. We also assumed that the gaseous flow rates $\mathbf{q}$ are measured on-line. It follows that Eq. (1) can be rephrased as:

$$
\begin{aligned}
& \dot{\boldsymbol{\xi}}_{a}=\mathrm{K}_{a} \mathbf{r}(\boldsymbol{\xi})+D\left(\boldsymbol{\xi}_{a}^{i n}-\boldsymbol{\xi}_{a}\right)-\mathbf{q}_{a}(\boldsymbol{\xi}) \\
& \dot{\boldsymbol{\xi}}_{b}=\mathrm{K}_{b} \mathbf{r}(\boldsymbol{\xi})+D\left(\boldsymbol{\xi}_{b}^{i n}-\boldsymbol{\xi}_{b}\right)-\mathbf{q}_{b}(\boldsymbol{\xi})
\end{aligned}
$$

where $\left(\mathrm{K}_{a}, \mathrm{~K}_{b}\right),\left(\boldsymbol{\xi}_{a}^{i n}, \boldsymbol{\xi}_{b}^{i n}\right)$ and $\left(\mathbf{q}_{a}, \mathbf{q}_{b}\right)$ are the induced partition of $\mathrm{K}, \boldsymbol{\xi}^{i n}$ and $\mathbf{q}$, respectively.

We now state the main Assumption.

Assumption 1. Matrix $\mathrm{K}_{a} \in \mathbb{R}^{\left(n_{\xi}-n_{b}\right) \times n_{r}}$ is a full rank column matrix (i.e. with $n_{\xi}-n_{b} \geq$ $\left.n_{r}\right)$.

If Assumption 1 holds, the following $n_{b}$-dimensional auxiliary system can be derived from a linear change of variables:

$$
\begin{aligned}
\dot{\mathbf{z}}= & -D\left(\mathbf{z}-\mathbf{z}^{i n}\right)-\mathrm{P} \mathbf{q}_{a}-\mathbf{q}_{b} \\
\boldsymbol{\xi}_{b}= & \mathbf{z}-\mathrm{P} \boldsymbol{\xi}_{a} \\
\text { where: } & \mathrm{P}:=-\mathrm{K}_{b} \mathrm{~K}_{a}^{\dagger}
\end{aligned}
$$


and $\mathrm{K}_{a}^{\dagger}$ is a left inverse of $\mathrm{K}_{a}$ (such an inverse is guaranteed to exist from Assumption 1). It should be noted that the measured state variables $\boldsymbol{\xi}_{a}$ play the role of inputs in the auxiliary system $(2,3)$. Note also that the auxiliary system is a consequence of both stoichiometric and mass-conservation arguments, and that it corresponds to the reaction invariants [12].

An asymptotic observer for the unmeasured variables is obtained by simply making a copy of Eqs. $(2,3)$, provided that a condition of persistence of excitation holds for the input variable $D$ [1]. Such asymptotic observers have however several known defects due to their open-loop nature. In particular, their derivation relies on the implicit assumption that the mass-balance part of the model is known perfectly and that neither the measurements, nor the values of the feeding inputs, nor the estimates of the yield coefficients (matrix K) are biased. Otherwise the predictions of the mass-balance-based observer would be corrupted and may provide very loose estimates of the true concentration values $\boldsymbol{\xi}_{b}$.

For large uncertainties in particular, it is no longer possible to build exact observers guaranteeing that the observation errors converges to zero. Therefore, the observation principle must be revisited and the results must be weakened. In the next section, we propose new probabilistic observers that estimate the PDF of the unknown state variables $\boldsymbol{\xi}_{b}$.

\section{PROBABILISTIC OBSERVERS}

As mentioned earlier in the paper, biological processes generally exhibit very large uncertainties in both the influent composition and the model parameters. This is particularly tricky for wastewater treatment processes where sensors that are capable of measuring the organic pollutant concentrations accurately and in real-time are still lacking. But although the influent composition is generally not known precisely, still some information is available for it in most practical applications. For the model parameters on the other hand, estimates are often obtained on application of a parameter identification procedure. PDFs (or at least good estimates of them) can therefore be known for either kinds of uncertainties. However, such PDFs can be of any type (especially for the influent concentrations), i.e., they are not necessarily normally distributed.

\subsection{Mathematical Background}

We consider continuous bioprocesses described by mass-balance-based bioprocess models of the form (1). For such models, major sources of uncertainties arise from:

- the unmeasured state variables at initial time $\boldsymbol{\xi}_{b 0}$;

- the unmeasured influent concentrations $\xi^{i n}$;

- the unknown model parameters $\mathbf{k}$ (e.g., the coefficients of the yield matrix K);

- the noise $\boldsymbol{\epsilon}$ in the measurements of the state variables $\boldsymbol{\xi}_{a}$ and of the gaseous flow rates $\mathbf{q}_{a}, \mathbf{q}_{b}$.

In the sequel, we make a distinction between the uncertainties originating from the initial conditions, and those corresponding to either influent concentrations, model parameters and measurement noises. These latter uncertainties are gathered within a unique vector $\mathbf{p}=\left(\boldsymbol{\xi}^{\text {in }}, \mathbf{k}, \boldsymbol{\epsilon}\right)$. 
In order to calculate the PDF of the unmeasured state variables $\boldsymbol{\xi}_{b}$, we consider the auxiliary linear dynamical system $(2,3)$ defined in section 2 . It is worth reminding that this system requires that Assumption 1 holds and results from a linear change of variables that utilizes the available state measurements $\boldsymbol{\xi}_{a}$ in order to eliminate the uncertain kinetic rates $\mathbf{r}(\boldsymbol{\xi})$. Here, we rewrite the auxiliary system $(2,3)$ in a somewhat more general form, thus yielding the following linear differential system:

$$
\left\{\begin{aligned}
\dot{\mathbf{z}}(t) & =\mathrm{A}(\mathbf{p}, t) \mathbf{z}(t)+\mathbf{b}(\mathbf{p}, t) \\
\boldsymbol{\xi}_{b}(t) & =\mathbf{z}(t)+\mathbf{c}(\mathbf{p}, t) \\
\mathbf{z}(0) & =\boldsymbol{\xi}_{b 0}-\mathbf{c}(\mathbf{p}, 0)
\end{aligned}\right.
$$

where $\mathbf{p} \in \mathcal{P} \subset \mathbb{R}^{n_{p}}$ denotes the vector of the uncertain parameters; $\boldsymbol{\xi}_{b 0} \in X_{0} \subset \mathbb{R}^{n_{b}}$ are the unknown initial conditions; and the coefficients of $\mathrm{A} \in \mathbb{R}^{n_{b} \times n_{b}}, \mathbf{b} \in \mathbb{R}^{n_{b}}$ and $\mathbf{c} \in \mathbb{R}^{n_{b}}$ are $\mathcal{C}^{1}$ functions of the parameters $\mathbf{p}$.

Assumption 2. The uncertain parameters $\mathbf{p}$ in system $(\mathcal{S})$ are constant with known PDFs (stationary disturbances). In addition, we suppose that the PDFs of $\mathbf{p}$ and of the initial conditions $\boldsymbol{\xi}_{\mathbf{0}}$ are independent.

It should be noted that Assumption 2, although not fundamentally necessary, considerably simplifies the synthesis of a probabilistic observer for $(\mathcal{S})$. In the more general case where $\mathbf{p}$ is piecewise constant however, all subsequent developments extend in a straightforward manner.

Notations 1. We use the following notations for the random variables: $\mathbf{X}_{0}=\left(\boldsymbol{\xi}_{b 0}, \mathbf{p}\right)$, $\mathrm{Z}_{0}=\left(\boldsymbol{z}_{0}, \mathbf{p}\right), \mathrm{X}_{t}=\left(\boldsymbol{\xi}_{b}(t), \mathbf{p}\right)$ and $\mathrm{Z}_{t}=(\boldsymbol{z}(t), \mathbf{p})$. In addition, we denote by $f_{\mathrm{X}_{0}}, f_{\mathrm{Z}_{0}}, f_{\mathrm{X}_{t}}$ and $f_{\mathrm{Z}_{t}}$ their respective PDFs. Likewise, the PDFs of the unknown state variable $\boldsymbol{\xi}_{b}$ at time $t$ is denoted by $f_{\boldsymbol{\xi}_{b t}}$.

From classical linear systems theory, the solution $\boldsymbol{\xi}_{b}$ of $(\mathcal{S})$ is given by:

$$
\boldsymbol{\xi}_{b}(t)=\widetilde{\psi}_{t} \circ \widetilde{\varphi}_{t} \circ \widetilde{\psi}_{0}^{-1}\left(\boldsymbol{\xi}_{b 0}\right)
$$

where $\widetilde{\psi}_{t}$ and $\widetilde{\varphi}_{t}$ are the linear diffeomorphisms defined as:

$$
\begin{aligned}
\widetilde{\psi}_{t}: \mathbb{R}^{n_{b}} & \longrightarrow \mathbb{R}^{n_{b}} \\
\mathbf{z} & \longmapsto \mathbf{z}+\mathbf{c}(\mathbf{p}, t),
\end{aligned}
$$

and

$$
\begin{aligned}
\widetilde{\varphi}_{t}: \mathbb{R}^{n_{b}} & \longrightarrow \mathbb{R}^{n_{b}} \\
\mathbf{z} & \longmapsto \Phi(\mathbf{p}, 0, t) \mathbf{z}+\int_{0}^{t} \Phi(\mathbf{p}, \tau, t) \mathbf{b}(\mathbf{p}, \tau) \mathrm{d} \tau .
\end{aligned}
$$

Here, $\Phi(\mathbf{p}, \cdot, \cdot) \in \mathbb{R}^{n_{b} \times n_{b}}$ denotes the transition matrix of $(\mathcal{S})$, i.e., the solution of the matrix differential equation:

$$
\begin{aligned}
\frac{\mathrm{d}}{\mathrm{d} t} \Phi(\mathbf{p}, \tau, t) & =\mathrm{A}(\mathbf{p}, t) \Phi(\mathbf{p}, \tau, t) \\
\Phi(\mathbf{p}, \tau, \tau) & =I_{n_{b} \times n_{b}} .
\end{aligned}
$$

For the calculation of the PDF of the unmeasured state variables $\boldsymbol{\xi}_{b}(t)$, the idea is to take advantage of the aforementioned analytical relationship between the initial condition $\boldsymbol{\xi}_{b 0}$, the parameters $\mathbf{p}$ and the available measurements (contained in both terms $\mathbf{b}(\mathbf{p}, t)$ and $\mathbf{c}(\mathbf{p}, t)$ ). This is formalized in the algorithm described in Proposition 1 below. 
Proposition 1. Under Assumption 2 and given the PDFs of the initial conditions, parameters and measurements, an estimate of PDF $f_{\boldsymbol{\xi}_{b t}}$ of the unmeasured state variables at a given time $t>0$ can be computed from the following three-step algorithm:

1. Estimate the PDF $f_{\mathrm{Z}_{0}}$ of the random variable $\mathrm{Z}_{0}$ at initial time, from the joint $P D F \mathrm{X}_{0}$ of both the unmeasured state variables $\boldsymbol{\xi}_{b 0}$ and the parameters $\mathbf{p}$.

2. Compute the image $f_{\mathrm{Z}_{t}}$, at time $t$, of the $P D F f_{\mathrm{Z}_{0}}$ by the flow of the differential equation $\dot{\mathbf{z}}=A \mathbf{z}+\mathbf{b}$ in the auxiliary system $(\mathcal{S})$.

3. Estimate the PDF $f_{\mathrm{X}_{t}}$ of the random variable $\mathrm{X}_{t}$, at time $t$, from the PDF of $\mathrm{Z}_{t}$.

Steps 1 and 3 rely on the diffeomorphisms $\psi_{0}^{-1}$ and $\psi_{t}$, respectively, with $\psi_{t}$ being defined as:

$$
\begin{aligned}
\psi_{t}: v_{t} & \longrightarrow \mathcal{U}_{t} \\
\mathrm{z}_{t} & \longmapsto \mathrm{X}_{t}=\left(\boldsymbol{\xi}_{b}(t)=\widetilde{\psi}_{t}(\mathbf{z}(t), \mathbf{p}), \mathbf{p}\right) .
\end{aligned}
$$

On the other hand, step 2 is based on the diffeomorphism $\varphi_{t}$ defined as:

$$
\begin{aligned}
\varphi_{t}: V_{0} & \longrightarrow \mathcal{V}_{t} \\
\mathrm{Z}_{0} & \longmapsto \mathrm{Z}_{t}=\left(\mathbf{z}(t)=\widetilde{\varphi}_{t}\left(\mathbf{z}_{0}, \mathbf{p}\right), \mathbf{p}\right) .
\end{aligned}
$$

Moreover, we have $\mathfrak{U}_{0} \supset \mathcal{X}_{0} \times \mathcal{P}, \mathcal{V}_{0}=\psi_{t}^{-1}\left(\mathcal{U}_{0}\right), \mathcal{V}_{t}=\varphi_{t}\left(\mathcal{V}_{0}\right)$ and $\mathcal{U}_{t}=\psi_{t}\left(\mathcal{V}_{t}\right)$.

Overall, the PDF $f_{\boldsymbol{\xi}_{b t}}$ corresponding to the image $\boldsymbol{\xi}_{b}(t)$ of the random variables $\boldsymbol{\xi}_{b_{0}} \in X_{0}$, for $\mathbf{p} \in \mathcal{P}$, is given by:

$$
f_{\boldsymbol{\xi}_{b_{t}}}(x)=\left\{\begin{array}{l}
\int_{\mathcal{P}} \frac{f_{\mathrm{X}_{0}}\left(\psi_{0} \circ \varphi_{t}^{-1} \circ \psi_{t}^{-1}(x, \mathbf{p})\right)}{\exp \left(\int_{0}^{t} \operatorname{Tr}[\mathrm{A}(\mathbf{p}, \tau)] \mathrm{d} \tau\right)} \mathrm{d} \mathbf{p} \quad \text { if } \quad x \in \psi_{t} \circ \varphi_{t} \circ \psi_{0}^{-1}\left(X_{0}, \mathcal{P}\right) \\
0 \quad \text { otherwise. }
\end{array}\right.
$$

Remark 1. In the particular case of a standard bioprocess, the matrix $\mathrm{A}$ is simply defined as: $\mathrm{A}(\mathbf{p}, t)=-D(t) I_{n_{b} \times n_{b}}-$ see Eq. (2). Accordingly, the following simplification holds:

$$
\operatorname{Tr}[\mathbf{A}(\mathbf{p}, t)]=-n_{b} \times D(t) .
$$

In order to prove Proposition 1, we first recall the following result (see, e.g., Corollary 4.7.4 in [18]), which provides a general framework for calculating the image of a given PDF by a $\mathcal{C}^{1}$-diffeomorphism.

Theorem 1. Let $\mathfrak{U}$ be a subset of $\mathbb{R}^{n}$, and let $\phi: \mathcal{U} \rightarrow \mathcal{V}=\phi(\mathfrak{U})$ be a $\mathcal{C}^{1}$ diffeomorphism. If $\mathrm{Z}$ is a random variable with a PDF $f_{\mathrm{Z}}$, then the random variable $\mathrm{W}=\phi(\mathrm{Z})$ has a PDF $f_{\mathrm{W}}$ given by:

$$
f_{\mathrm{W}}(\omega)=\left\{\begin{array}{l}
f_{\mathrm{Z}}\left(\phi^{-1}(\omega)\right) \operatorname{det}\left(\mathcal{J}_{\phi^{-1}}(\omega)\right) \quad \text { if } \omega \in \mathcal{V} \\
0 \quad \text { otherwise }
\end{array}\right.
$$

where $\mathcal{J}_{\phi}$ denotes the Jacobian matrix of $\phi$.

Proof of Proposition 1. It is clear, from the analytical solution of $(\mathcal{S})$, that $\varphi_{t}$ and $\psi_{t}$ are $\mathcal{C}^{1}$-diffeomorphisms. Therefore, the overall transformation $\psi_{0} \circ \varphi_{t}^{-1} \circ \psi_{t}^{-1}$ is itself a $\mathcal{C}^{1}$ diffeomorphism (chain rule of differentiation), and Theorem 1 applies. Because,

$$
\operatorname{det} \mathcal{J}_{\psi_{0}}=\operatorname{det} \mathcal{\partial}_{\psi_{t}}=I_{n_{b}+n_{p}}
$$


then,

$$
\operatorname{det} \partial_{\left(\psi_{0} \circ \varphi_{t}^{-1} \circ \psi_{t}^{-1}\right)}=\operatorname{det} \partial_{\varphi_{t}^{-1}} .
$$

Moreover,

$$
\operatorname{det} \partial_{\varphi_{t}}=\operatorname{det}\left(\begin{array}{cc}
\Phi(\mathbf{p}, 0, t) & \star \\
0_{n_{p} \times n_{b}} & I_{n_{p} \times n_{p}}
\end{array}\right)=\operatorname{det} \Phi(\mathbf{p}, 0, t)
$$

and from the Jacobi-Liouville formula, we obtain

$$
\operatorname{det} \mathcal{\partial}_{\varphi_{t}}=\exp \left(\int_{0}^{t} \operatorname{Tr}[\mathbf{A}(\mathbf{p}, \tau)] \mathrm{d} \tau\right) .
$$

Finally, the PDF of the unmeasured state variables $\boldsymbol{\xi}_{b}$ is obtained, at any time $t>0$, by integrating the joint state/parameter $\mathrm{PDF}$ with respect to the uncertain parameters $\mathbf{p} \in \mathcal{P}$, which completes the proof.

\subsection{Numerical Implementation and Practical Application}

The numerical implementation of the previous framework can be decomposed into two consecutive steps:

1. Compute the image $f_{\mathrm{X}_{t}}$ of the joint PDF $f_{\mathrm{X}_{0}}$ by considering the diffeomorphism $\psi_{0} \circ \varphi_{t}^{-1} \circ \psi_{t}^{-1}$ in Proposition 1. This can be done, e.g., by defining a grid in the joint state/parameter space. Note that due to the linearity of the auxiliary system $(\mathcal{S})$ with respect to $\mathbf{z}$, one only needs to calculate the trajectories of $(\mathcal{S})$ at the vertices of the variation domain of $\mathbf{z}$; the intermediate values are then easily calculated from linear interpolation.

2. Integrate the joint state/parameter $\mathrm{PDF} f_{\mathrm{X}_{t}}$, at a given time $t$, with respect to the parameters $\mathbf{p} \in \mathcal{P}$ in order to obtain an estimate of the PDF $f_{\boldsymbol{\xi}_{b t}}$ of the unmeasured state variables $\boldsymbol{\xi}_{b}(t)$.

Mention should be made at this point that step 2 is by far the most complex and computationally demanding step in the numerical procedure. Because the influence of the initial condition $\boldsymbol{\xi}_{\mathbf{0}}$ vanishes as time increases, the joint PDF tends to become degenerate (it becomes infinitely thin), hence giving rise to serious numerical issues. Particular attention must therefore be payed to the integration of the joint PDF on $\mathcal{P}$ (see Eq. (4)) in order to guarantee accurate results. These issues are illustrated in Example 1 below. Different approaches are then discussed in subsection 3.3, which allow one to address these problems.

Example 1. An illustration of the practical difficulties that are encountered for the numerical implementation of the probabilistic observers is provided through the following simple differential system:

$$
\left\{\begin{aligned}
\dot{z}(t) & =-z(t)+p \quad \forall t>0, \\
z(0) & =z_{0} .
\end{aligned}\right.
$$

We assume that both PDFs of $z_{0}$ and $p$ are Gaussian distributions, denoted by $\mathcal{N}\left(\overline{z_{0}}, \sigma_{z_{0}}\right)$ and $\mathcal{N}\left(\bar{p}, \sigma_{p}\right)$, respectively. Then, on application of Proposition 1, the joint PDF $f_{\mathrm{Z}_{t}}$ of the random variable $Z_{t}=(z(t), p)$, at a given time $t>0$, is given by:

$$
f_{\mathrm{Z}_{t}}(\omega)=\frac{f_{\mathrm{Z}_{0}}\left(\varphi_{t}^{-1}(\omega)\right)}{\exp (-t)}
$$


for all $\omega=(y, q) \in \mathbb{R}^{2}$, where

$$
\begin{aligned}
f_{\mathrm{Z}_{0}}(\omega) & =\frac{1}{2 \pi \sigma_{z_{0}} \sigma_{p}} \mathrm{e}^{-\frac{1}{2}\left[\left(\frac{y-\overline{z_{0}}}{\sigma z_{0}}\right)^{2}+\left(\frac{q-\bar{p}}{\sigma_{p}}\right)^{2}\right]} \\
\varphi_{t}^{-1}(\omega) & =\left(\begin{array}{c}
\mathrm{e}^{t} y+\left(1-\mathrm{e}^{t}\right) q \\
q
\end{array}\right) .
\end{aligned}
$$

By combining these expressions and letting $t$ go to infinity, it can be easily checked that:

$$
\begin{array}{lll}
\lim _{t \rightarrow+\infty} f_{\mathrm{Z}_{t}}(\omega)=+\infty & \text { if } y=q, \\
\lim _{t \rightarrow+\infty} f_{\mathrm{Z}_{t}}(\omega)=0 & \text { otherwise. }
\end{array}
$$

This is illustrated in Fig. 1.

\subsection{Practical Measures to Avoid Degenerate PDFs}

We start this subsection by noting that the case of interest in this work corresponds to matrix A being Hurwitz, because $(\mathcal{S})$ is intended to be used as an auxiliary system/observer. In this case, the influence of the uncertain initial conditions $\boldsymbol{\xi}_{b 0}$ progressively vanishes with time, thus eventually resulting in a degenerate PDF (see Example 1 above for an illustration). In fact, the diffeomorphism $\widetilde{\varphi}_{t}$ itself becomes degenerate for asymptotically stable differential systems, in the sense that it is no longer invertible asymptotically, i.e.,

$$
\lim _{t \rightarrow+\infty}\|\Phi(\mathbf{p}, 0, t)\|=0
$$

with $\|\cdot\|$ being a matrix norm.

In order to avoid such a situation in practice, we consider a small threshold value $\eta>0$. Then, for a given $\eta$,

$$
\exists t_{\eta}>0 \text { such that }\|\Phi(\mathbf{p}, 0, t)\|<\eta \quad \forall t>t_{\eta} .
$$

For those problems verifying $n_{p} \geq n_{b}$, we give the following Proposition that defines an approximate diffeomorphism of $\varphi_{t}$.

Proposition 2. Let $\varepsilon$ be a small positive constant, and assume there exists a time $t_{\eta}$ such that $\left|\varphi_{t}\left(\mathbf{Z}_{0}\right)-\varphi_{t}^{\infty}\left(\mathbf{p}^{\prime}\right)\right| \leq \varepsilon$ for any initial PDF $\mathbf{Z}_{0} \in \mathcal{V}_{0}$, where $\varphi_{t}^{\infty}$ is a diffeomorphism from $\mathcal{P}^{\prime}$ (resulting from an appropriate projection of $\mathcal{P}$ onto $\mathbb{R}^{n_{b}}$ ) to $\mathbb{R}^{n_{b}}$. Then, the solution $\boldsymbol{\xi}_{b}(t)$ of $(\mathcal{S})$ can be approximated as:

$$
\boldsymbol{\xi}_{b}(t) \approx \widetilde{\psi}_{t} \circ \varphi_{t}^{\infty}\left(\mathbf{p}^{\prime}\right) \quad \forall t>t_{\eta} .
$$

Remark 2. This proposition is in fact quite general, and the hypotheses are naturally verified for many practical problems. In the case where they are not satisfied, one may still be able to find an appropriate reduced diffeomorphism either by considering a subset of the components of $\varphi_{t}$ only, or by viewing $\varphi_{t}$ as the projection of another diffeomorphism from a space of higher dimension.

By construction, the reduced diffeomorphism $\varphi_{t}^{\infty}$ is simpler than $\varphi_{t}$ for it only depends on a reduced number of components of the uncertain parameters $\mathbf{p}$, and provides an appropriate 
approximation of $\varphi_{t}$ for $t>t_{\eta}$. From a more practical point of view, $\varphi_{t}^{\infty}$ is indeed trivial for many applications, as it is the case for instance in Example 1 (see below).

At this point, it becomes clear that a similar procedure as the one described in Proposition 1 can be utilized to calculate the PDF $f_{\boldsymbol{\xi}_{b}}$, based on the reduced diffeomorphisms $\varphi_{t}^{\infty}$ and $\widetilde{\psi}_{t}$. Here, the algorithmic procedure is reduced to steps 1 and 2 only, and one finally needs to integrate the joint PDF of $\mathbf{X}_{t}=\left(\boldsymbol{\xi}_{b}(t), \mathbf{p}^{\prime}\right)$ on the reduced parameter space $\mathcal{P}^{\prime}$ in order to obtain the desired PDF $f_{\boldsymbol{\xi}_{b t}}$.

Example 1 (continued). On application of Proposition 2, the following trivial diffeomorphism is obtained (the same notation as in Example 1 are used):

$$
\varphi_{t}^{\infty}(q)=q .
$$

Accordingly, the following approximation is obtained for the PDF of $z(t)$ :

$$
f_{z_{t}}(q)=f_{p}\left(\varphi_{t}^{\infty-1}(q)\right)=f_{p}(q) \quad \forall q \in \mathbb{R}
$$

Note that the PDF of $z$ is then equal to the PDF of $p$.

An alternative approach that may be used to prevent the $\operatorname{PDF} f_{\boldsymbol{\xi}_{b t}}$ from becoming degenerate consists of adding an artificial term that acts as a diffusion [19]. A simple way of doing it is to replace the transition matrix $\Phi(0, t, \mathbf{p})$ in the expression of $\widetilde{\varphi}_{t}$ by $\left[\Phi(0, t, \mathbf{p})+\eta \times I_{n_{b} \times n_{b}}\right]$, with $\eta \ll 1$. This alternative approach also presents the advantage for the user to keep the same numerical procedure for all $t>0$.

In the coming practical case study, note that we do not have to deal with the aforementioned numerical issues. This is because the PDF of the state estimates does not become degenerate within the considered time frame, as the observer is reinitialized on a weekly basis by using new off-line measurements.

\section{INDUSTRIAL CASE STUDY}

In this section, we illustrate our approach with the concrete case study of an industrial anaerobic digestion (AD) wastewater treatment plant. AD consists of several consecutive biological degradation steps that take place in the absence of oxygen, each step being performed by different groups of specialized bacteria. Complex organic compounds are decomposed and bioconverted, on the one hand, into biogas (i.e., a mixture of $\mathrm{CO}_{2}$ and $\mathrm{CH}_{4}$ ) and, on the other hand, into microbial biomass and residual organic matter.

The main advantages of AD wastewater treatment plants, as compared to the more classical aerobic activated sludge plants, are their ability to handle substrates that are otherwise difficult to degrade at high concentrations, their high efficiency, as well as their low sludge production. Moreover, the produced methane can be utilized as a valuable energy resource. However, many industrials are still reluctant to use AD processes, mainly because AD processes have two stable equilibrium points [20], one of which corresponds to biomass washout and must be avoided for obvious reasons. Accordingly, AD processes therefore require a strong monitoring procedure in order to operate safely. Moreover, the observer's robustness towards modeling uncertainties (kinetic rates in particular) is of the utmost importance for $\mathrm{AD}$, since still no reliable model has been proposed to date. 


\subsection{Mass-Balance Modeling of the Digester}

We use a very simple model that describes the key features of AD [21]. Note that this model only takes the main degradation processes into account and is therefore a rough summary of the overall biological reaction network. It accounts for a single substrate (index of the total organic matter concentration, called Chemical Oxygen Demand (COD)) and a single bacterial population, denoted by $s$ and $x$, respectively. By assuming perfect mixing in the digester, the following 2-dimensional dynamical model is obtained:

$$
\begin{aligned}
& \dot{x}(t)=-\alpha D(t) x(t)+\mu(\cdot) x(t) \\
& \dot{s}(t)=-D(t)\left(s(t)-s^{i n}(t)\right)-k_{1} \mu(\cdot) x(t)
\end{aligned}
$$

where $s^{i n}(t)$ stands for the concentration of substrate in the feeding stream; $k_{1}$ is the yield coefficient associated to biomass production; and $\alpha$ represents the fraction of bacteria being present in the liquid phase (the remaining fraction $(1-\alpha)$ is attached onto a support within the digester). In addition, the methane flow rate $q_{\mathrm{CH}_{4}}$ is defined as follows:

$$
q_{\mathrm{CH}_{4}}(t)=k_{2} \mu(\cdot) x(t)
$$

where $k_{2}$ stands for the yield coefficient associated to methane production. It should be noted that the bacterial growth rate, $\mu(\cdot)$, is often described as a non-monotonic function of Haldanetype [21] that is responsible for the process instability.

\subsection{Probabilistic Observer for the COD Concentration}

The objective in this application is to design an observer capable of estimating the COD concentration $s$, based on the methane measurements, and without making any assumption on the growth rate function $\mu(\cdot)$. Also, we want to take advantage of the off-line COD measurements that are generally performed at a very low sampling frequency, e.g., once a week. In practice, the observer will therefore run in open-loop mode between two consecutive COD measurements, and will then be reinitialized after a new COD measurement has become available (see subsection 3.3).

Based on the model equations (5)-(7), the following auxiliary system can be derived for the biomass concentration, provided that the methane gaseous flow rate as well as the influent COD are measured on-line (see section 2):

$$
\dot{s}(t)=-D(t) s(t)+\left[D(t) s^{i n}-\frac{k_{1}}{k_{2}} q_{\mathrm{CH}_{4}}(t)\right] .
$$

A set of experimental data obtained from a 2,000 $\mathrm{m}^{3}$ industrial digester located at Agralco Ltd. in Estella, Spain, is considered subsequently. This digester is fed with wine distillery vinasses, and measurements are available for the dilution rate $D$ and for the methane outflow rate $q_{\mathrm{CH}_{4}}$, both at a high sampling frequency and for more than 140 days.

In order to design a probabilistic observer that estimates the COD concentration based on Eq. (8), the following uncertainties are taken into account:

i) Parameter $\gamma=\frac{k_{1}}{k_{2}}$. The value of $\gamma$ was estimated off-line based on mass-balance considerations along with its standard deviation: $\gamma=5.3010^{-2} \pm 0.98 \%$. It is assumed that the probability density for this parameter is Gaussian. 
ii) Influent concentration $s^{i n}$. The uncertainty on $s^{i n}$ is handled by considering a multiplicative noise $\varepsilon$ defined as $s^{i n}(t)=\overline{s^{i n}}(1+\varepsilon(t)), \forall t$. It was found from experimental data that $\overline{s^{i n}}=35.9 \mathrm{~g} \cdot \mathrm{L}^{-1}$ and that the PDF of $\varepsilon$ can be modeled by a normal distribution $\mathcal{N}\left(0, \sigma_{\varepsilon}\right)$ with $\sigma_{\varepsilon}=0.124$.

iii) measured COD concentration $s^{m e s}$. The observer is reinitialized on a weekly basis by using the available off-line COD measurements. The uncertainty associated to the COD measurements is modeled by a uniform PDF (within the range $s^{m e s} \pm 20 \%$ ).

On the other hand, the measurements of $D$ and $q_{\mathrm{CH}_{4}}$, which are obtained at a very high sampling frequency, have been low-pass filtered and, for the sake of simplicity, no error is taken into account for these measurements.

A probabilistic observer for the COD concentration is derived from Eq. (8) on application of Proposition 1. In this case, note that $\psi_{t}=\psi_{0}^{-1}=I$ and $\Phi(\tau, t)=\exp \left[-\int_{\tau}^{t} D(s) \mathrm{d} s\right]$. The resulting PDFs of the estimate $\hat{s}$ are represented in Figure 2 (upper plot) at different time instants. For the sake of observer validation, experimental COD concentrations that were measured on a daily basis are also displayed on this figure; it is worth reminding to the reader that these additional points were not used for designing the probabilistic observer. Note that the variation range of $\hat{s}$ (corresponding to the vertical segments) is rather conservative, but a significant part of the range has zero probability. Also note that most of the experimental points are enclosed within the non-zero probability part, and that the maximum likelihood estimate is close to the experimental points.

A quite straightforward utilization of the resulting PDFs of $\hat{s}$ consists of extracting their maximum likelihood values (or their average values) as well as their standard deviations. In practice, it is very likely that only these two informations will be made available to the user. Both are shown in figure 2 (lower plot). It can be seen from this plot that the estimates are close to the experimental measurements and that the trajectories corresponding to the standard deviation of $\hat{s}$ mainly enclose these measurements. However, the fact that the data are not in the appropriate range between days 43 to 49.5 warrants some further explanations. In fact the off-line measurements of the COD concentration turn out to be corrupted due to chemicals expiration. Interestingly enough, it appears that the probabilistic observer could therefore be used in such situations for efficiently detecting measurement biases or failures.

The interested reader is also referred to [22] for discussion of different application of the probabilistic observers in the field of bioprocesses.

\section{PRACTICAL CONSIDERATIONS}

The proposed probabilistic observers present some peculiarities in comparison to usual observers. The most important, maybe, lies in the fact that they compute, at any time instant, the exact $\mathrm{PDF}$ of the estimated state variables. Accordingly, there is no required convergence period regarding the use of these observers. What can be said concerning convergence, however, is that the estimated state PDF converges to a limit PDF that is independent of the initial state $\mathrm{PDF}$, provided that matrix $\mathrm{A}$ in $(\mathcal{S})$ is Hurwitz; moreover, the corresponding rate of convergence is dictated by the smallest eigenvalue of matrix $A$. This "natural" convergence is closely related to the problem of degenerate PDFs discussed earlier in subsection 3.3. Therefore, 
probabilistic observers could be equally well seen as detectors, since they are fully based on the (reduced) dynamics of the system. Moreover, these observers are much more efficient when an accurate estimate of the initial state PDF is provided, since convergence to the aforementioned limit PDF is then much faster. This was illustrated by the industrial case study presented previously.

In practice, the information contained in the calculated state PDFs is often too complex to be displayed for an industrial operator. Instead, the end-user can be given the average value together with an estimate of the standard deviation value. An interval enclosing those state values having a cumulated probability equal to a given confidence level (e.g. 95\%) can be also be easily calculated.

On the other hand, the information contained in the state PDF can be fed directly into a supervision procedure [23], hence allowing the supervision system to account for the level of uncertainty when making a decision regarding the state of the process. This is of crucial importance in certain practical applications having multiple equilibrium points, such as the anaerobic digestion process. The ability to detect, early enough during the operation, potential state trajectories outside of the attraction basin of the desired operating point could indeed help prevent the process from dramatic problems.

Finally, since many applications in biotechnology are characterized by a high level of noise, probabilistic observers may produce very widespread PDFs that are useless from a practical point of view. However, determining which source of uncertainty must be reduced in order for the observer to produce a more accurate prediction is quite straightforward. Therefore, the proposed observers could be used as tools to identify which measurements should be improved in priority for producing more accurate state estimates. In such situation, specific effort could then be made on the designated sensors.

\section{CONCLUSIONS}

In this paper, the design of probabilistic observers has been investigated for a class of continuous biological processes described by mass-balance-based models. It has been shown how the PDF of the unmeasured state variables can be obtained from the knowledge of the PDF of the uncertain parameters, inputs and measurement noises. The practical implementation of probabilistic observers has also been closely considered. These observers share some common features with both asymptotic observers and classical Kalman filtering techniques, but also differ in several points; the main features of probabilistic observers are summarized hereafter.

- in order to combat the inherent uncertainties characterizing biological systems, probabilistic observers are based on a reduced system obtained by eliminating a number of functions (e.g., microbial kinetics) based on linear algebra manipulations;

- in line with asymptotic observers [1], probabilistic observers are open-loop by nature, in the sense that the estimation is not corrected by the difference between the predicted outputs and the measurements;

- unlike classical Kalman filters finally, probabilistic observers can handle any kind of PDF, including uniform and normal PDFs as well as compact support based PDFs characterizing bounded uncertainties. 
The design and application of a probabilistic observer for the estimation of the COD concentration in an industrial anaerobic wastewater treatment plant has been presented. This case study demonstrates both the tractability and the good performances of the proposed observers for a practical problem. Finally, a number of general considerations have been discussed regarding potential applications of probabilistic observers in connection to supervision and fault diagnosis of bioprocesses.

\section{ACKNOWLEDGEMENTS}

This work has been carried out with the support provided by the European Commission, Information Society Technologies program, Key action I Systems \& Services for the Citizen, contract TELEMAC number IST-2000-28256. This information is provided under the sole responsibility of the author and does not necessarily represent the opinion of the European Commission, which is not responsible for any use that might be made of it.

\section{REFERENCES}

1. G. Bastin and D. Dochain. On-line estimation and adaptive control of bioreactors. Elsevier, Amsterdam, The Netherlands, 1990.

2. O. Bernard and J.-L. Gouzé. State estimation for bioprocesses. In A. Agrachev, editor, ICTP Lecture Notes, pages 813-855, Trieste, Italy, 2002.

3. J.-P. Gauthier, H. Hammouri, and F. Celle. A simple observer for nonlinear systems - Applications to bioreactors. IEEE Trans. Automat. Control., 37:875-880, 1992.

4. J.-P. Gauthier and I. A. Kupka. Observability and observers for nonlinear systems. SIAM J. Contr. Optim., 32(4):975-994, 1994.

5. F. Deza, D. Bossanne, E. Busvelle, J.-P. Gauthier, and D. Rakotopara. Exponential observers for nonlinear systems. IEEE Trans. Autom. Contr., 38(3):482-484, 1993.

6. E. Busvelle and J.-P. Gauthier. High-gain and non-high-gain observers for nonlinear systems. In A. Anzaldo-Meneses, F. Monroy-Pérez, B. Bonnard, and J. P. Gauthier, editors, Contemporary Trends in Nonlinear Geometric Control Theory and its Applications, pages 233-256, London, 2002. World Scientific.

7. O. Bernard, G. Sallet, and A. Sciandra. Nonlinear observers for a class of biological systems. Application to validation of a phytoplanktonic growth model. IEEE Trans. Automat. Control., 43:1056-1065, 1998.

8. H. J. Sussmann and P. V. Kokotovic. The peaking phenomenon and the global stabilization of nonlinear systems. IEEE Trans. Autom. Contr., 36:424-440, 1991.

9. G. Bastin and J. van Impe. Nonlinear and adaptive control in biotechnology: a tutorial. European Journal of Control, 1(1):1-37, 1995.

10. M. Fjeld. On a pitfall in stability analysis of chemical reactions. Chem. Eng. Sci., 23: 565-573, 1968 .

11. O. A. Asbjørnsen and M. Fjeld. Response modes of continuous stirred tank reactors. Chem. Eng. Sci., 25:1627-1636, 1970

12. O. A. Asbjørnsen. Reaction invariants in the control of continuous chemical reactors. Chem. Eng. Sci., 27:709-717, 1972

13. P. Bogaerts. A hybrid asymptotic-kalman observer for bioprocesses. Bioprocess and Biosystems Engineering, 3:249-255, 1999.

14. V. Lemesle and J.-L. Gouzé. An hybrid bounded error observer for uncertain bioreactor models. Bioprocess and Biosystems Engineering,27:311-318,2005.

15. J.-L. Gouzé, A. Rapaport, and Z. Hadj-Sadok. Interval observers for uncertain biological systems. Ecological Modelling, 133:45-56, 2000.

16. A. Rapaport and J.-L. Gouzé. Parallelotopic and practical observers for non-linear uncertain systems. International Journal of Control, 76(3):237-251, 2003.

17. J.-L. Gouzé, A. Rapaport, and M. Z. Hadj-Sadok. Interval observers for uncertain biological systems. Ecological Modelling, 133:45-56, 2000.

18. G. R. Grimmett and D. R. Stirzaker. Probability and Random Processes. Oxford University Press Inc., New York, 3rd edition edition, 2001.

19. E.C. Zeeman. Stability of dynamical systems. Nonlinearity, 1:115-155, 1988. 
20. O. Bernard, Z. Hadj-Sadok, D. Dochain, A. Genovesi, and J.-P. Steyer. Dynamical model development and parameter identification for an anaerobic wastewater treatment process. Biotechnol. Bioeng., 75(4):424438, 2001.

21. L. Mailleret, O. Bernard, and J.-P. Steyer. Robust nonlinear adaptive control for bioreactors with unknown kinetics. Automatica, 40(8):365-383, 2004

22. B. Chachuat, and O. Bernard. Design of probabilitic observers for mass-balance based bioprocess models. 9th Int. Symp. on Computer Applications in Biotechnology, March 28th-31st 2004, Nancy, France.

23. J. Chen and R. Patton Robust model-based fault diagnosis for dynamic systems . Kluwer Academic Publishers, Boston, MA, 1999. 


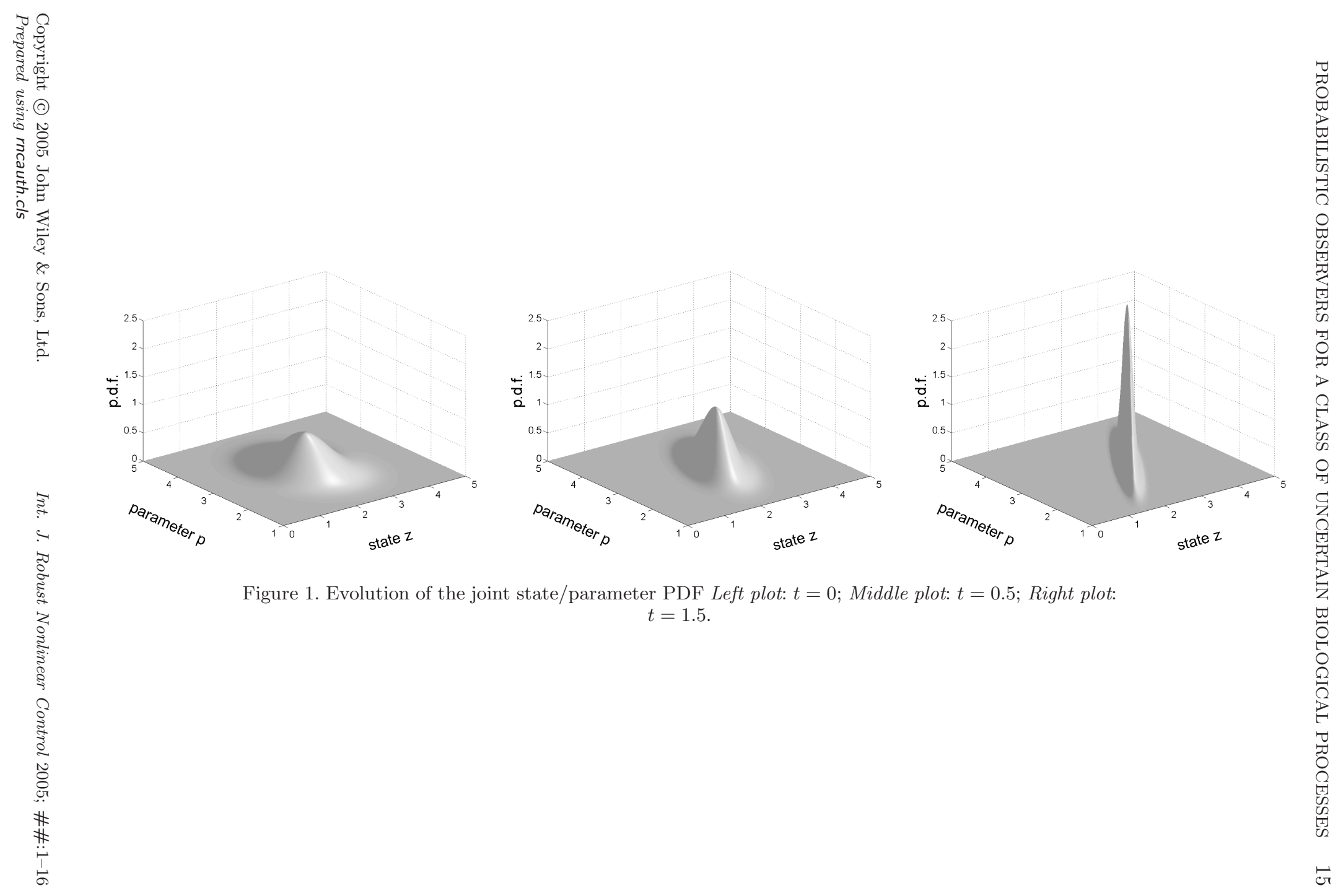



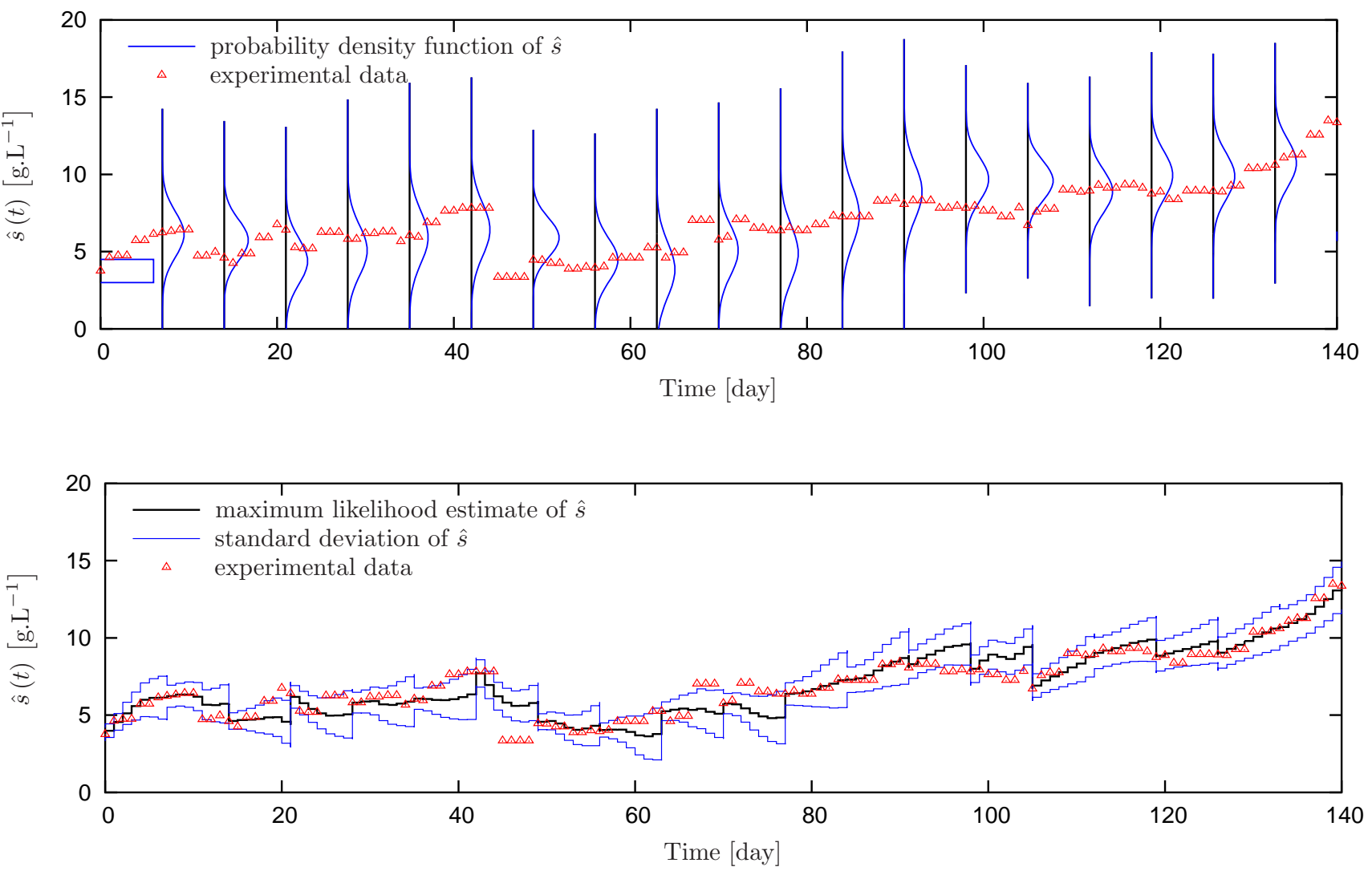

Figure 2. Estimated COD concentration in the digester. Top plot: Probability density functions. Bottom plot: Maximum likelihood and standard deviation. 